\section{Impacto de la Artritis Reumatoide sobre la \\ funcionalidad física y la calidad de vida relacionada con la salud: Metaanálisis 2003-2013}

\section{Resumen}

Introducción: La artritis reumatoide es una enfermedad que afecta la funcionalidad física y la calidad de vida relacionada con la salud (CVRS); en la literatura médica se presenta una elevada heterogeneidad sobre el impacto de la enfermedad en estos dominios.

Objetivo: Evaluar el impacto de la artritis reumatoide sobre la funcionalidad física y la CVRS a partir de estudios publicados entre 2003 y 2013. Materiales y métodos: Revisión sistemática con metaanálisis. Se realizó una búsqueda en cuatro bases de datos multidsicplinarias con los términos de búsqueda "calidad de vida \& artritis reumatoide" y sus homólogos en inglés y portugués. Se evaluó la calidad de los artículos por dos investigadores garantizando la exhaustividad y reproducibilidad. La funcionalidad física se evaluó con el HAQ-DI (Health Assesment Questionnaire Disability Index) y la CVRS con el MOSSF-36 (Medical Outcome Study Short Form 36). El análisis se basó en frecuencias, medidas de resumen, intervalos de confianza y prueba $\mathrm{t}$ Student para los análisis de heterogeneidad y sensibilidad.

Resultados: Se incluyeron 48 investigaciones en 27 países, en los cuales se evaluó la funcionalidad física en 24.237 pacientes y la calidad de vida en 46.725. Se obtuvo un promedio ponderado de $1,12 \pm 0,8$ en el HAQ-DI y en el MOSSF-36 fue 35,2 26,3 para el componente físico y 47,3 $\pm 6,9$ para el mental, lo que resulta menor a los puntajes establecidos para poblaciones sanas de 50 puntos.

Conclusión: La artritis reumatoide impacta negativamente la CVRS, siendo mayor su efecto sobre el componente físico, mientras que la funcionalidad física se afecta moderadamente; este conocimiento resulta útil para direccionar los lineamientos de intervención en el individuo con la enfermedad e investigación en esta área.
John Querubín

Franco-Aguirre', Jaiberth Antonio

Cardona-Arias ${ }^{2}$

1 Microbiólogo y Bioanalista. cMSc Microbiología y Bioanálisis. Docente Escuela de Microbiología, Universidad de Antioquia. Medellín, Colombia. Investigador Grupo Salud y Sostenibilidad.

2 Microbiólogo y Bioanalista, MSc Epidemiología. Universidad de Antioquia UdeA, Calle 70 No. 5221, Medellín, Colombia. Facultad de Medicina Universidad Cooperativa de Colombia. Medellín, Colombia.

\section{Correspondencia:}

Jaiberth Antonio Cardona Arias

” jaiberthcardona@gmail.com 
Palabras claves: Artritis reumatoide, Calidad de vida, Aptitud física, Revisión

\section{Impact of Rheumatoid Arthritis on physical function and health related quality of life: Meta-Analysis 2003-2013}

\section{Abstract}

Background: rheumatoid arthritis is a disease that affects physical function and the health related quality of life (HRQL), in the literature is presented high heterogeneity about the impact of the disease in these domains.

Objective: Evaluate the impact of rheumatoid arthritis on physical function and HRQOL from studies published between 2003 and 2013. Methods: Systematic review with meta-analysis. A search was conducted on four multidisciplinary data bases with search terms "quality of life \& rheumatoid arthritis" and their counterparts in Spanish and Portuguese were performed. The quality of articles was evaluated by two researchers to ensure the completeness and reproducibility. Physical function was assessed with the HAQ-DI (Health Assessment Questionnaire Disability Index) and HRQOL with MOSSF-36 (Medical Outcome Study Short Form 36. The analysis was based on frequencies, summary statistics, confidence intervals and Student $t$ test for heterogeneity and sensitivity analysis.

Results: 48 studies in 27 countries were included, in which the physical function and quality of life was assessed in 24,237 and 46,725 patients respectively. A weighted average of $1.12 \pm 0.8$ was obtained in the HAQ-DI and the MOSSF-36 was $35.2 \pm 6.3$ for the physical component and $47.3 \pm 6.9$ for the mental, which is lower than the scores established for healthy populations of 50 points.

Conclusion: Rheumatoid arthritis negatively impacts $H R Q L$, being greater its effect on the physical component, whereas physical functioning is affected moderately, this knowledge is useful to address the guidelines for intervention in the individual with the disease and research in this area.

Keywords: Arthritis, Rheumatoid; Quality of Life, Physical Fitness, Review. 


\section{Introducción}

La artritis reumatoide es una enfermedad con una amplia distribución geográfica y una prevalencia estimada entre el 0,5 y 1,0\% de la población adulta mundial, ésta varía según factores como la raza, la etnia y condiciones ambientales(1,2); es así como su ocurrencia en nativos americanos está por encima del 3\% (2,3), por debajo del $0,2 \%$ en algunas poblaciones de África y Asia (2), alrededor del 0,61\% en ciudades y del $0,16 \%$ en zonas rurales (4-6). En general, afecta dos veces más a la mujer que al hombre y en su etiología se han sugerido diversos factores como los genéticos y agentes infecciosos, sin que se tenga hasta el momento una explicación concluyente sobre su causa $(1,6)$.

Clínicamente se trata de una enfermedad autoinmune caracterizada por ser un proceso inflamatorio crónico, progresivo e incapacitante de las articulaciones, cuyas manifestaciones incluyen tumefacción y dolor articular, rigidez matutina, fatiga y reducción de la movilidad, las cuales afectan la funcionalidad física y social del individuo, incrementa el estrés psicológico y las incapacidades laborales, lo que en conjunto deteriora la calidad de vida $(7,8)$.

Dado el deterioro de la calidad de vida del individuo afectado con artritis reumatoide, se ha recomendado que la evaluación y seguimiento de la enfermedad incluya la valoración de la funcionalidad física y de la calidad de vida relacionada con la salud (CVRS) (9). La primera se describe como la capacidad del individuo para desempeñar correctamente actividades básicas e instrumentales de la vida cotidiana (10), mientras que la segunda es entendida como el grado de satisfacción que el individuo tiene con su bienestar físico, emocional y social, y que está condicionada por la presencia de la enfermedad $(11,12)$. Para la evaluación de la CVRS y la funcionalidad física se han empleado escalas o instrumentos cuyas propiedades de validez, confiabilidad y sensibilidad al cambio deben verificarse para garantizar su adecuada y correcta aplicación (9). Además de ello, debe tenerse en cuenta su clasificación de acuerdo a la población objetivo, en este sentido, se han diferenciado dos tipos de instrumentos, los genéricos y los específicos (13).

Los instrumentos genéricos se caracterizan por poder ser aplicados en población general y en grupos específicos de pacientes, permitiendo comparaciones entre población enferma y sana (13), entre éstos se destacan el Medical Outcome Study Short Form 36 (MOS-SF36), WhoQol Bref, Nottingham Health Profile (NPH), EuroQol 5D ( EQ-5D) y el Health Assesment Questionnaire Disability Index (HAQ-DI); como escalas aplicadas en investigaciones sobre CVRS y funcionalidad física en artritis reumatoide (14). Las escalas específicas incluyen dimensiones de CVRS concretas para la enfermedad o grupo de pacientes por lo que presentan mayor sensibilidad clínica (13), en este grupo sobresale la aplicación de instrumentos como el Reumathoid Arthritis Quality of Life (RAQOL) y el Arthritis Impact measurement Scales (AIMS) (14).

El uso individual o combinado de estos instrumentos ha estado supeditado por las características de la población a estudiar, los objetivos perseguidos (clínicos, investigativos o financieros), el contenido de la escala, su disponibilidad en el contexto sociocultural y las dimensiones que cada uno incluye para abordar el constructo de CVRS (13). En el caso de la funcionalidad física un elemento determinante en la elección del instrumento lo constituyen los diferentes aspectos de la cotidianidad del individuo que están relacionados con su desempeño físico (10).

En este contexto el MOSSF-36 y el HAQ-DI se han constituido como instrumentos de amplia aplicación en la investigación clínica y epidemiológica de la artritis reumatoide $(15,16)$ dada la temporalidad de su concepción (HAQ en la década de los 80 y el MOS-SF36 principios de los 90) $(15,17)$ aunado al excelente comportamiento de sus propiedades psicométricas en estudios de validación, destacándose la validez de criterio y de constructo, la confiabilidad en términos de consistencia interna y reproducibili- 
dad (18-23), la sensibilidad al cambio, traducciones en gran cantidad de idiomas y adaptaciones transculturales $(15,16)$, ofreciendo una alta disponibilidad en diferentes partes del mundo.

Sumado a estas características, el estudio de la CVRS y de la funcionalidad física en artritis reumatoide se ha favorecido por la confluencia de factores y situaciones inherentes a la enfermedad como son su cronicidad, su amplia distribución geográfica, el desarrollo de nuevos esquemas y terapias farmacológicas que impactan la funcionalidad física y otras dimensiones de la CVRS del individuo, el creciente interés en estos temas por parte de instituciones del área de la salud y tomadores de decisiones, la necesidad de trascender las mediciones clínicas y la relevancia de incluir este tipo de mediciones para la definición de estrategias de intervención social y distribución de recursos $(24,25)$.

Dado el anterior contexto, en la literatura científica se pueden encontrar numerosos estudios que reportan diferentes grados de impacto de la enfermedad en la funcionalidad física y la CVRS del individuo, es así como los estudios de Rojas et al y Wallenius et al $(26,27)$ reportan puntajes indicativos de una funcionalidad física levemente afectada, en contraste, los resultados de Nagappa et al y kojima et al $(28,29)$ evidencian puntajes sugestivos de una funcionalidad gravemente afectada, de forma similar los estudios de Yacoub et al, Mota et al, Nicassio et al, Ovayolu et al y Akar et al (30-34), exponen resultados que demuestran importantes diferencias relacionadas con la dimensión de la CVRS mayormente afectada y su grado de afectación.

Estas diferencias entre los resultados de cada estudio, ponen en manifiesto la necesidad de agrupar de manera sistemática los puntajes de las mediciones de la CVRS y la funcionalidad física de los estudios individuales de acuerdo a las dimensiones del MOSSF-36 y el HAQ-DI, con los objetivos de definir el grado de impacto de la funcionalidad física y de las dimensiones de CVRS en el individuo con artritis reumatoide y consolidar la hipótesis sobre la dimensión o componente más afectado, como medio para la orientación de acciones en salud.

En razón de lo anterior, se diseñó una investigación con el objetivo de evaluar el impacto de la artritis reumatoide sobre la funcionalidad física y la CVRS a partir de estudios publicados entre 2003 y 2013.

\section{Materiales y métodos}

\section{Tipo de estudio}

Revisión sistemática con meta-análisis

\section{Instrumentos de medición de la funcionalidad física y de la CVRS}

El HAQ-DI es un cuestionario que consta de 20 preguntas sobre actividades de la vida cotidiana, el cual evalúa los movimientos finos de miembros superiores e inferiores. Se divide en 8 categorías que incluyen vestirse, levantarse, comer, caminar, higiene personal, el alcance y agarre de objetos, y otras actividades. Cada pregunta dispone de 4 posibilidades de respuesta calificadas de 0 a 3, el primero indica ausencia de dificultad y el segundo imposibilidad de realizar la actividad durante la última semana. El puntaje global resulta del promedio de las 8 categorías, el cual puede variar entre 0 (sin discapacidad) y 3 (grave discapacidad) (15).

El MOSSF-36 es un cuestionario de 36 preguntas que mide 8 dimensiones relacionadas con la CVRS: función física, limitaciones del rol causadas por problemas físicos, dolor corporal, percepción de la salud general, función social, limitaciones del rol causadas por problemas emocionales; salud mental y vitalidad. Las cuatro primeras resumen el componente físico del instrumento en tanto que las restantes resumen el componente mental, además existe un elemento no incluido en estas ocho categorías que explora los cambios experimentados en el estado de salud en el último año. La puntuación del 
MOSSF-36 varía en un rango de 0 (peor estado) a 100 (mejor estado) y un valor de referencia para los componentes físico y mental establecido en población sana de 50ะ10 $(16,35,36)$.

\section{Protocolo de investigación}

La búsqueda y selección de los artículos se realizó con base en la declaración PRISMA (Preferred Reporting Items for Systematic reviews and MetaAnalyses), la cual establece cuatro fases en la ejecución de revisiones sistemáticas y metaanálisis: identificación, tamización, elección e inclusión (37). Identificación: Se realizó una búsqueda por sensibilidad de la literatura relacionada con CVRS en artritis reumatoide en las bases de datos Pubmed, Science direct, Lilacs y Scielo utilizando las estrategias de búsqueda "quality of life \& rheumatoid arthritis", "calidad de vida \& artritis reumatoide" y "qualidade de vida \& artrite reumatoide". Los artículos obtenidos en esta fase fueron exportados al programa EndnoteWeb para la eliminación de duplicados.

\section{Tamización}

Los estudios identificados fueron tamizados a partir de la aplicación de los siguientes criterios de inclusión: i) término de búsqueda en el título, resumen y/o en las palabras clave ii) publicados entre enero de 2003 y diciembre de 2013 y iii) artículos originales.

\section{Elección}

Los que cumplieron los criterios de inclusión fueron leídos en su totalidad para aplicar los siguientes criterios de exclusión, i) los que no hicieron explícito el instrumento de medición de la CVRS, ii) cuyo objetivo principal era la validación de un instrumento o la medición de CVRS como un desenlace secundario de una intervención, iii) aquellos en los que no se reportó el puntaje de la funcionalidad física y CVRS según el HAQ-DI y el MOSSF-36 respectivamente, y iv) artículos con problemas de validez interna por no hacer control de sesgos.

\section{Inclusión}

A los artículos restantes se les realizó síntesis cualitativa y cuantitativa de los resultados.

\section{Recolección de la información}

Para garantizar la exhaustividad del protocolo de investigación se realizó una búsqueda por sensibilidad, sin circunscribirla a términos DeCS (Descriptores de Ciencias de la Salud) o MeSH (Medical Subject Headings), esto permitió la obtención de un mayor número de estudios frente a la búsqueda por especificidad. La aplicación del protocolo de investigación se llevó a cabo por dos investigadores de forma independiente para garantizar la reproducibilidad de la revisión, las discrepancias se resolvieron por consenso y referencia a un tercero. La extracción de la información se realizó con base en un protocolo y se almacenó en una base de datos diseñada en Excel, realizada por dos investigadores con el fin de garantizar la reproducibilidad inter-observador de la información recolectada y analizada, en este proceso se hallaron índices Kappa de 1,00 para las variables año de publicación, país y tipo de población, esto corresponde a una concordancia perfecta en la escala de Landis y Koch; mientras que para las variables edad, años de diagnóstico, puntajes de función física, CVRS y actividad inflamatoria se hallaron coeficientes de correlación intraclase de 1,00. Los puntajes de actividad inflamatoria, se tomaron de acuerdo a los instrumentos clinimétricos: DAS-28 (Disease Activity Score), SDAI (Simplified Disease Activity Index) y CDAI (Clinical Disease Activity Index) cuyos valores indican diferentes grados de actividad. En este sentido valores mayores a 5,1, 40 y 22 en el DAS-28, SDAI y CDAl respectivamente, indican enfermedad muy activa, entre 3,2 y 5,1, 20 y 40, 10 y 22 son indicadores de actividad moderada y menores a 3,2, 20 y 10 indicarían baja o leve actividad inflamatoria de la artritis reumatoide (9).

\section{Análisis de la información}

Se calcularon puntajes promedio ponderados del HAQ-DI y de los componentes mental (función físi- 
ca, limitaciones de rol causadas por problemas físicos; dolor corporal y salud general) y físico (función social, limitaciones de rol causadas por problemas emocionales, salud mental y vitalidad) del MOSSF-36 mediante el estadístico $\bar{X}=\sum_{i=1}^{n} \frac{X_{i} \cdot n_{i}}{n}$, acompañados de su desviación estándar; para los estudios que no reportaron la medida de dispersión se realizó imputación con el método hot-deck mediante estratificación según el país de publicación (38).

Para el análisis de heterogeneidad de los puntajes del HAQ-DI y del MOSSF-36 se calcularon intervalos de confianza para la media a través del estadístico IC95\% $=\bar{X} \pm Z \frac{\alpha}{2} \cdot\left(\frac{D S}{\sqrt{n}}\right)$ y con sus valores se realizaron Forest Plot para cada variable de respuesta. Posteriormente se hizo un análisis de sensibilidad para explorar la influencia de cada estudio sobre el puntaje global, mediante el cálculo de la media ponderada resultante de la exclusión de cada estu- dio y su comparación con la media ponderada total (incluyendo su intervalo de confianza) mediante la prueba t-student para muestras independientes.

Los análisis se realizaron con la hoja de cálculo de Microsoft Excel 2010 y el programa para análisis epidemiológico de datos tabulados de la Organización Panamericana de la Salud (Epidat) versión 3.0 con una significación de 0,05.

\section{Resultados}

En la búsqueda inicial se identificaron 3.195 artículos, al aplicar los criterios de inclusión se redujeron a 255 artículos y posterior a la aplicación de los criterios de exclusión 48 artículos fueron tomados para la síntesis cualitativa y cuantitativa de la revisión (Figura 1).

Las investigaciones se desarrollaron en 27 países e incluyeron 24.237 pacientes en la evaluación de la

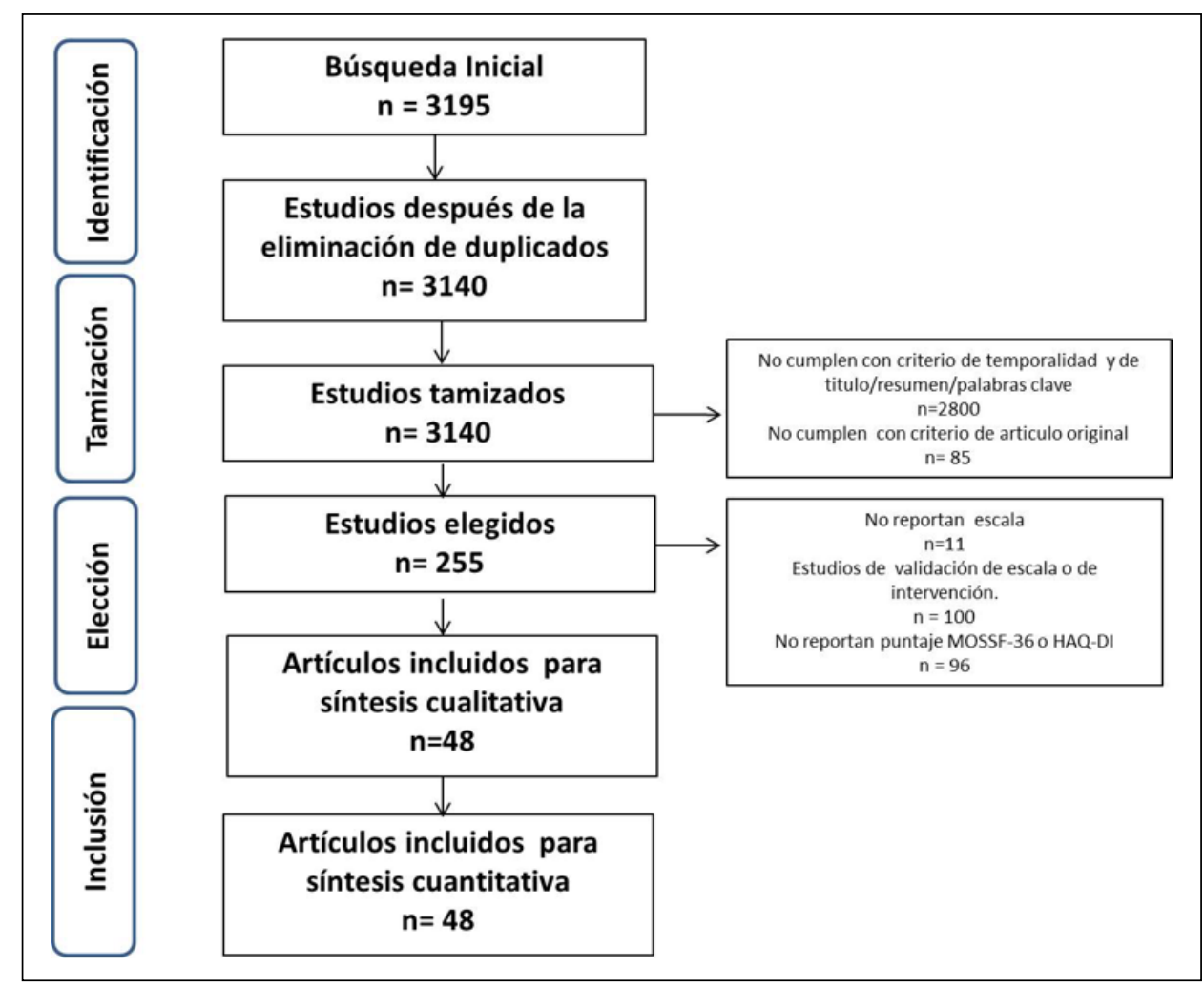

Figura 1. Algoritmo de selección de artículos 
funcionalidad física y 46.725 para la CVRS. Entre las características sociodemográficas se observó que el $70,5 \%$ de la población pertenecía al género femenino y el rango de edad estuvo entre 36 y 63 años, los años de diagnóstico de la enfermedad variaron en un rango de 0,6 a 18,8 años y el promedio de la actividad inflamatoria de la enfermedad basada en el DAS-28 varió entre 3,4 y 5,9 (Tabla 1).

Del total de artículos incluidos, 13 evaluaron la funcionalidad física con el HAQ-DI, 19 evaluaron

Tabla 1. Características sociodemográficas y clínicas de las poblaciones estudiadas

\begin{tabular}{|c|c|c|c|c|c|c|c|}
\hline $\begin{array}{l}\text { MOSSF-36 } \\
\text { y HAQ DI }\end{array}$ & Año & Lugar & $\mathbf{N}^{\circ}$ & $\begin{array}{c}\mathrm{N}^{\circ} \\
\text { mujeres }\end{array}$ & Edad (DE) & $\begin{array}{c}\text { Años de } \\
\text { diagnóstico } \\
\text { (DE) }\end{array}$ & $\begin{array}{l}\text { Actividad } \\
\text { inflamatoria } \\
\text { (DE) }\end{array}$ \\
\hline Akar $S^{34}$ & 2008 & Turquía & 44 & 44 & $55,2(9,6)$ & $8,9(9,6)$ & $3,9(1,4)^{*}$ \\
\hline Corbacho $\mathrm{Ml}^{39}$ & 2010 & Brasil & 53 & 47 & $51,9(12)$ & $9,8(7,2)$ & $4,7(1,8)^{*}$ \\
\hline Courvoisier DS 40 & 2012 & Suiza & 15282 & 10467 & $52,3(14)$ & $9,9(9,7)$ & NR \\
\hline Eser $\mathrm{F}^{41}$ & 2012 & Turquía & 150 & 126 & $53,2(12,1)$ & $12,3(8,3)$ & $3,4(1,3)^{*}$ \\
\hline Gronning $\mathrm{K}^{42}$ & 2010 & Noruega & 310 & 223 & $50,2(11,6)$ & NR & $4,6(1,1)^{*}$ \\
\hline Hodkinson $B^{43}$ & 2012 & Sudáfrica & 171 & 140 & $47(12,5)$ & $1(0,6)$ & $38,4(16,2)^{\star *}$ \\
\hline Jorge $\mathrm{RT}^{44}$ & 2010 & Brasil & 43 & 36 & 51,6 & 12,2 & NR \\
\hline Kojima $\mathrm{M}^{29}$ & 2009 & Japón & 120 & 98 & $57,7(12,8)$ & $11,4(10,1)$ & NR \\
\hline Mota LM LM $^{31}$ & 2012 & Brasil & 65 & 56 & $45,6(14,5)$ & $0,6(0,35)$ & NR \\
\hline Nicassio PM $^{32}$ & 2011 & $\begin{array}{l}\text { Estados } \\
\text { Unidos }\end{array}$ & 106 & 88 & $56,2(12,4)$ & $12(11,4)$ & $4,3(0,9)$ \\
\hline Radner $\mathrm{H}^{45}$ & 2011 & Austria & 380 & 306 & $60,7(13,2)$ & $11,7(10,1)$ & $9,08(8,6) * * *$ \\
\hline Tang B46 & 2009 & $\begin{array}{l}\text { Estados } \\
\text { Unidos }\end{array}$ & 2200 & 1584 & 49,6 & 12,4 & $N R$ \\
\hline Thyberg $\left.\right|^{47}$ & 2005 & Suecia & 202 & 137 & $55(15)$ & NR & NR \\
\hline Wallenius $\mathrm{M}^{27}$ & 2009 & Noruega & 474 & 372 & $36(6,1)$ & $5,6(5,8)$ & $4,3(1,4)^{\star}$ \\
\hline Yacoub $\mathrm{Yl}^{48}$ & 2011 & Marruecos & 185 & 185 & $46,6(10,8)$ & $10,6(8,1)$ & $5,4(1,4)^{*}$ \\
\hline Yacoub Y|30 & 2012 & Marruecos & 255 & 181 & $46,6(11,6)$ & $12,5(7,5)$ & $5,9(1,8)^{*}$ \\
\hline Subtotal & & & 20040 & 14090 & & & \\
\hline \multicolumn{8}{|c|}{ HAQ DI } \\
\hline Blaizot $A^{49}$ & 2013 & Francia & 73 & 55 & $60,2(11,9)$ & $15,2(9,6)$ & $3,8(1,4)^{*}$ \\
\hline Carreño $A^{50}$ & 2011 & España & 244 & 185 & $57,8(13,3)$ & $11(8,0)$ & $N R$ \\
\hline Garip Y51 & 2011 & Turquía & 153 & 129 & $53,4(12)$ & $12,2(8,2)$ & $3,4(1,4)^{*}$ \\
\hline Haroon N52 & 2007 & India & 136 & 117 & $44,4(10,5)$ & $9(5,8)$ & $4,4(1,4)^{\star}$ \\
\hline Helland $Y^{53}$ & 2008 & Noruega & 830 & 614 & $58,5(14,2)$ & $13,4(10,3)$ & NR \\
\hline Khera $\mathrm{K}^{54}$ & 2010 & India & 166 & NR & NR & $10(3,1)$ & $4,2(1,1)^{*}$ \\
\hline Kobelt $G^{55}$ & 2008 & Francia & 1487 & 1294 & $62,7(12,5)$ & $18(11,3)$ & $4,4(2,3)^{*}$ \\
\hline Marra $\mathrm{CA}^{56}$ & 2004 & Canadá & 309 & 243 & $61,5(25,9)$ & $13,9(11,4)$ & NR \\
\hline Nagappa AN28 & 2012 & India & 159 & NR & $47,8(12,5)$ & NR & $4,9(0,4)^{*}$ \\
\hline Pentek $\mathrm{M}^{57}$ & 2008 & Hungría & 255 & NR & $55,5(12,3)$ & $9,0(9,3)$ & $5,1(1,4)^{\star}$ \\
\hline
\end{tabular}




\begin{tabular}{|c|c|c|c|c|c|c|c|}
\hline & Año & Lugar & $\mathbf{N}^{\circ}$ & $\begin{array}{c}\mathbf{N}^{\circ} \\
\text { mujeres }\end{array}$ & Edad (DE) & $\begin{array}{l}\text { Años de } \\
\text { diagnóstico } \\
\text { (DE) }\end{array}$ & $\begin{array}{c}\text { Actividad } \\
\text { inflamatoria } \\
\text { (DE) }\end{array}$ \\
\hline \multicolumn{8}{|c|}{ HAQ DI } \\
\hline Rojas $A^{26}$ & 2009 & Colombia & 95 & 79 & $52,5(12,4)$ & $9,0(7,1)$ & $3,7(1,5)^{*}$ \\
\hline Standfield L58 & 2010 & Australia & 170 & 126 & $59,1(12,3)$ & $15,4(12,1)$ & $3,4(1,5)^{\star}$ \\
\hline Tang $\mathrm{CH}^{59}$ & 2013 & Taiwán & 120 & 92 & 54,5 & $8,2(6,1)$ & NR \\
\hline Subtotal & & & 4197 & & & & \\
\hline \multicolumn{8}{|c|}{ MOSSF-36 } \\
\hline Birtane $\mathrm{M}^{60}$ & 2007 & Turquía & 35 & NR & $49,4(10,4)$ & NR & NR \\
\hline Garcia-Poma 61 & 2007 & Perú & 359 & 312 & $58(14)$ & $16,5(10,8)$ & $4,9(1,5)^{*}$ \\
\hline He DY62 & 2012 & China & 250 & 156 & NR & $N R$ & $N R$ \\
\hline Ho RC 63 & 2011 & Singapur & 100 & 75 & $53,7(13,6)$ & $6,2(5,5)$ & $3,9(0,9)^{*}$ \\
\hline Kanecki K ${ }^{64}$ & 2013 & Polonia & 51 & 42 & $62,5(12,6)$ & NR & NR \\
\hline Kavati $A^{65}$ & 2013 & $\begin{array}{l}\text { Estados } \\
\text { unidos }\end{array}$ & 20432 & 16347 & $59,8(11,8)$ & NR & NR \\
\hline Kothe $\mathrm{R}^{66}$ & 2007 & Alemania & 150 & 87 & $56,6(13,5)$ & $18,8(11,2)$ & NR \\
\hline Lempp $H^{67}$ & 2011 & Inglaterra & 902 & 651 & $56,9(13)$ & NR & NR \\
\hline Ovayolu N ${ }^{33}$ & 2011 & Turquía & 264 & 190 & $20-65$ & $6,6(5,5)$ & NR \\
\hline Picavet $\mathrm{HS}^{68}$ & 2004 & Holanda & 156 & NR & $N R$ & NR & NR \\
\hline Piccinni $A^{69}$ & 2006 & Italia & 92 & 67 & 61,4 & 5 & NR \\
\hline Rupp $1^{70}$ & 2004 & Holanda & 490 & 356 & $60(13,4)$ & $10,7(9,2)$ & $3,5(1,3)^{\star}$ \\
\hline Salaffi $F^{71}$ & 2009 & Italia & 693 & 630 & $53,9(12,9)$ & $9,8(8,2)$ & $N R$ \\
\hline Salaffi $F^{72}$ & 2009 & Italia & 469 & 338 & $57,5(14,3)$ & $6,1(4,2)$ & $4,5(0,8)^{\star}$ \\
\hline Tander $\mathrm{B}^{73}$ & 2008 & Turquía & 30 & NR & $39,1(1,4)$ & NR & NR \\
\hline Tang B74 & 2009 & $\begin{array}{l}\text { Estados } \\
\text { unidos }\end{array}$ & 2000 & 1548 & 51,6 & NR & NR \\
\hline Vinaccia $S^{75}$ & 2006 & Colombia & 82 & 65 & $59(8,7)$ & $6,9(4,1)$ & NR \\
\hline West $E^{76}$ & 2005 & Suecia & 79 & 57 & 52,4 & $9,8(8,2)$ & NR \\
\hline West $E^{77}$ & 2009 & Suecia & 51 & 34 & 49,8 & 1 & NR \\
\hline Subtotal & & & 26685 & & & & \\
\hline
\end{tabular}

* DAS $28, * *$ SDAl, $* * *$ CDAI

la CVRS con el MOSSF-36 y 16 aplicaron ambos instrumentos. En las investigaciones que emplearon el HAQ-DI se obtuvo un promedio ponderado de $1,12 \pm 0,8$ con un rango entre 0,5 y 2,3 puntos; en los puntajes de los estudios individuales se observó una alta heterogeneidad (Figura 2).

Los promedios globales ponderados para los componentes físico y mental del MOSSF-36 fueron 35,2 $\pm 6,3$ (rango $=26,5-48,0)$ y $47,3 \pm 6,9$ (rango $=$
$36,2-55,2)$ respectivamente; en ambas dimensiones se halló heterogeneidad para los puntajes de CVRS de los estudios incluidos (Figura 3) y al comparar los puntajes de los dos componentes se halló una diferencia de 12,10 (IC 95\% =12,01- 12,19).

En el análisis de sensibilidad del HAQ-DI sólo se halló un cambio estadísticamente significativo con la exclusión del estudio de Courvoisier et al (40), para los demás estudios el cambio frente a la medida 


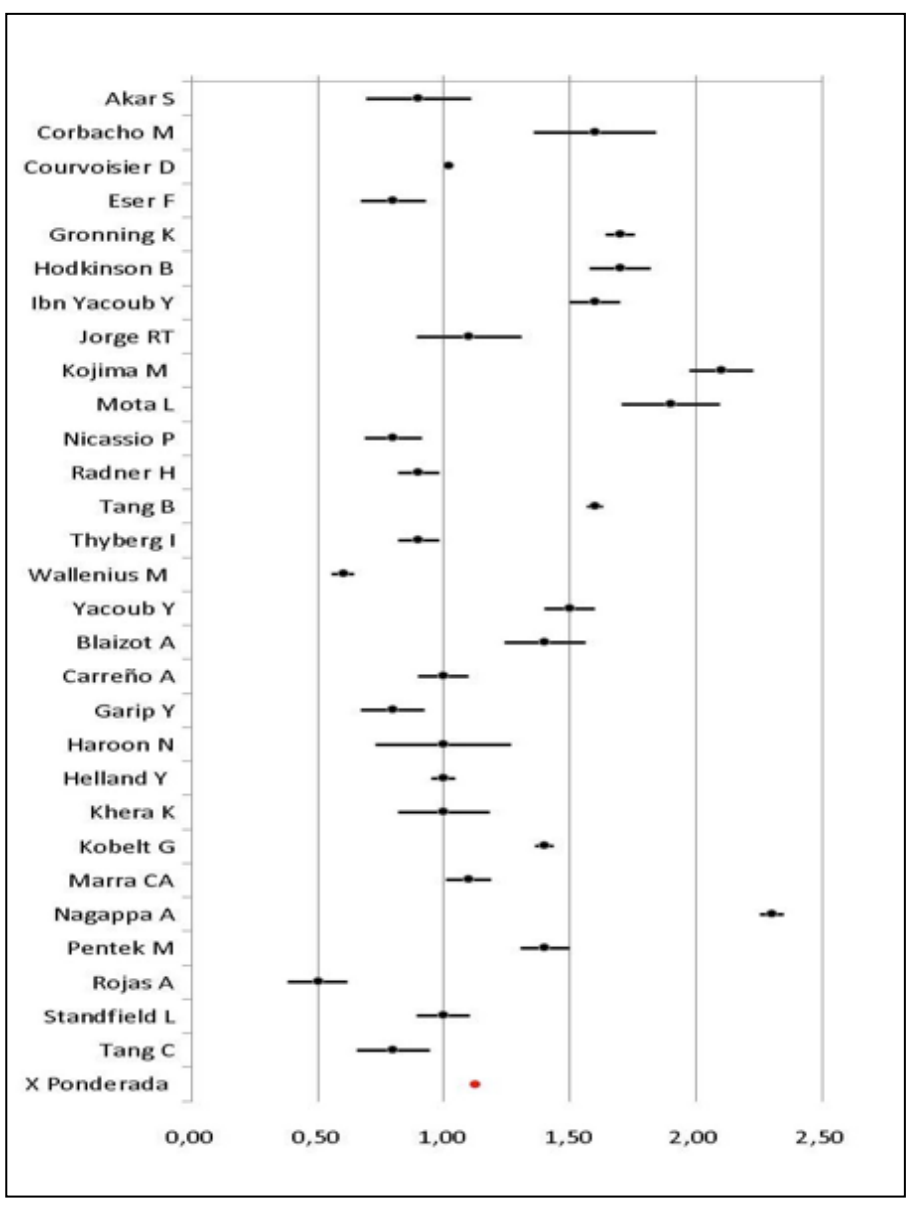

Figura 2. Análisis de la funcionalidad física según el HAQ-DI.

global fue de cero; demostrando la pertinencia de la medida combinada para la funcionalidad física. Respecto a la sensibilidad de los promedios globales obtenidos para los componentes físico y mental del MOSSF-36, se observaron cambios estadísticamente significativos con la exclusión de los estudios de Kavati et al (65) Lempp et al (67) Tang et al $(46,74)$ y Thyberg et al (47) (Tabla 2); sin embargo tales cambios no afectaron la conclusión hallada con la medida global expuesta dado que a pesar de ser estadísticamente significativos, en la escala de cero a 100 son clínicamente insignificantes (78).

\section{Discusión}

El presente estudio evidencia el creciente interés de investigadores por abordar la CVRS y funcionalidad

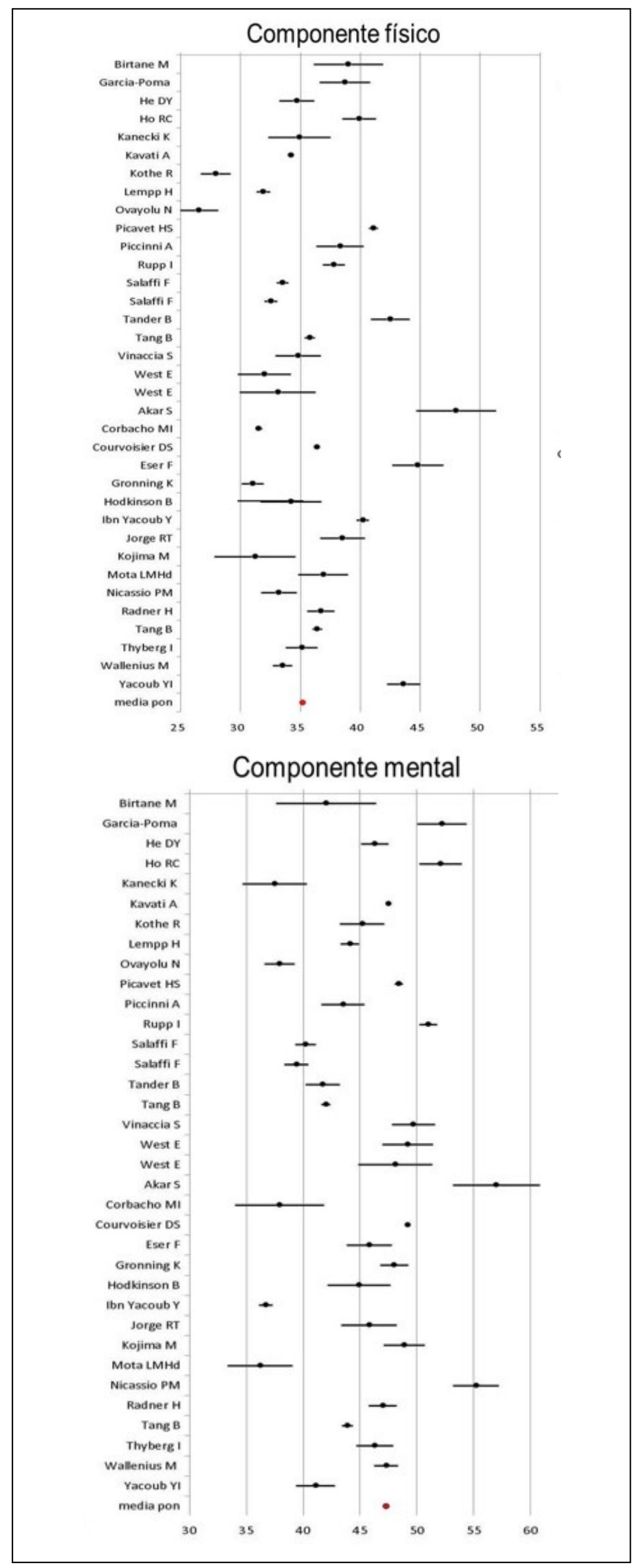

Figura 3. Análisis de la CVRS según el MOSSF-36. 
Tabla 2. Análisis de sensibilidad para puntajes ponderados del MOSSF36.

\begin{tabular}{|c|c|c|c|c|}
\hline \multirow{2}{*}{ ESTUDIO } & \multicolumn{2}{|c|}{ Físico } & \multicolumn{2}{|c|}{ Mental } \\
\hline & Media & Cambio & Media & Cambio \\
\hline Akar $\mathrm{S}^{34}$ & 35,2 & $0(-0,08 ; 0,08)$ & 47.3 & $0(-0,09 ; 0,09)$ \\
\hline Corbacho $\mathrm{Ml}^{39}$ & 35,2 & $0(-0,08 ; 0,08)$ & 47.3 & $0(-0,09 ; 0,09)$ \\
\hline Courvoisier DS 40 & 34,6 & $-0.6^{*}(-0,67 ;-0,53)$ & 46.4 & $-0.9 *(-0,98 ;-0,82)$ \\
\hline Eser $\mathrm{F}^{41}$ & 35,2 & $0(-0,08 ; 0,08)$ & 47.3 & $0(-0,09 ; 0,09)$ \\
\hline Gronning $\mathrm{K}^{42}$ & 35,2 & $0(-0,08 ; 0,08)$ & 47.3 & $0(-0,09 ; 0,09)$ \\
\hline Hodkinson $\mathrm{B}^{43}$ & 35,2 & $0(-0,08 ; 0,08)$ & 47.3 & $0(-0,09 ; 0,09)$ \\
\hline Jorge $\mathrm{RT}^{44}$ & 35,2 & $0(-0,08 ; 0,08)$ & 47.3 & $0(-0,09 ; 0,09)$ \\
\hline Kojima M²9 & 35,2 & $0(-0,08 ; 0,08)$ & 47.3 & $0(-0,09 ; 0,09)$ \\
\hline Mota LM $^{31}$ & 35,2 & $0(-0,08 ; 0,08)$ & 47.3 & $0(-0,09 ; 0,09)$ \\
\hline Nicassio PM³2 & 35,2 & $0(-0,08 ; 0,08)$ & 47.3 & $0(-0,09 ; 0,09)$ \\
\hline Radner $\mathrm{H}^{45}$ & 35,2 & $0(-0,08 ; 0,08)$ & 47.3 & $0(-0,09 ; 0,09)$ \\
\hline Tang B 46 & 35,1 & $-0.1 *(-0,18 ;-0,02)$ & 47.5 & $0.2^{*}(0,11 ; 0,29)$ \\
\hline Thyberg $1^{47}$ & 35,1 & $-0.1^{*}(-0,18-0,02)$ & 47.3 & $0(-0,09 ; 0,09)$ \\
\hline Ibn Yacoub $Y^{48}$ & 35,2 & $0(-0,08 ; 0,08)$ & 47.3 & $0(-0,09 ; 0,09)$ \\
\hline Ibn Yacoub Y30 & 35,2 & $0(-0,08 ; 0,08)$ & 47.3 & $0(-0,09 ; 0,09)$ \\
\hline Wallenius $\mathrm{M}^{27}$ & 35,2 & $0(-0,08 ; 0,08)$ & 47.3 & $0(-0,09 ; 0,09)$ \\
\hline Birtane $\mathrm{M}^{58}$ & 35,2 & $0(-0,08 ; 0,08)$ & 47.3 & $0(-0,09 ; 0,09)$ \\
\hline Garcia-Poma ${ }^{59}$ & 35,2 & $0(-0,08 ; 0,08)$ & 47.3 & $0(-0,09 ; 0,09)$ \\
\hline He DY60 & 35,2 & $0(-0,08 ; 0,08)$ & 47.3 & $0(-0,09 ; 0,09)$ \\
\hline Ho RC 61 & 35,2 & $0(-0,08 ; 0,08)$ & 47.3 & $0(-0,09 ; 0,09)$ \\
\hline Kanecki K ${ }^{62}$ & 35,2 & $0(-0,08 ; 0,08)$ & 47.3 & $0(-0,09 ; 0,09)$ \\
\hline Kavati $A^{65}$ & 36 & $0.8^{*}(0,66 ; 0,936)$ & 47.1 & $-0.2^{*}(-0,35 ;-0,05)$ \\
\hline Kothe $\mathrm{R}^{66}$ & 35.2 & $0(-0,08 ; 0,08)$ & 47.3 & $0(-0,09 ; 0,09)$ \\
\hline Lempp $H^{67}$ & 35,3 & $0,1(0,02 ; 0,18)$ & 47.4 & $0.1 *(0,01 ; 0,19)$ \\
\hline Ovayolu N ${ }^{31}$ & 35,3 & $0,1(0,02 ; 0,18)$ & 47.3 & $0(-0,09 ; 0,09)$ \\
\hline Picavet HS ${ }^{68}$ & 35,2 & $0 *(-0,08 ; 0,08)$ & 47.3 & $0 *(-0,09 ; 0,09)$ \\
\hline Piccinni $A^{69}$ & 35,2 & $0(-0,08 ; 0,08)$ & 47.3 & $0(-0,09 ; 0,09)$ \\
\hline Rupp $\left.\right|^{70}$ & 35,2 & $0(-0,08 ; 0,08)$ & 47.2 & $-0.1(-0,01 ; 0,19)$ \\
\hline Salaffi $F^{71}$ & 35,2 & $0(-0,08 ; 0,08)$ & 47.4 & $0.1(0,01 ; 0,19)$ \\
\hline Salaffi $F^{72}$ & 35,2 & $0(-0,08 ; 0,08)$ & 47.4 & $0.1(0,01 ; 0,19)$ \\
\hline Tander $\mathrm{B}^{73}$ & 35,2 & $0(-0,08 ; 0,08)$ & 47.3 & $0 *(-0,09 ; 0,09)$ \\
\hline Tang B B4 & 35,2 & $0 *(-0,08 ; 0,08)$ & 47.5 & $0.2 *(0,11 ; 0,29)$ \\
\hline Vinaccia $S^{75}$ & 35,2 & $0(-0,08 ; 0,08)$ & 47.3 & $0(-0,09 ; 0,09)$ \\
\hline West $\mathrm{E}^{76}$ & 35,2 & $0(-0,08 ; 0,08)$ & 47.3 & $0(-0,09 ; 0,09)$ \\
\hline West $E^{77}$ & 35,2 & $0(-0,08 ; 0,08)$ & 47.3 & $0(-0,09 ; 0,09)$ \\
\hline
\end{tabular}

*Se hallaron cambios estadísticamente significativos en el 0,01 
física en artritis reumatoide dado el aumento gradual de las investigaciones en los últimos 10 años y el amplio de número de países en los que se ha investigado. A su vez revela una importante afectación de la CVRS y la funcionalidad física en una numerosa y representativa población afectada por dicha enfermedad. Los puntajes de 35,2 y 47,3 para los componentes físico y mental del MOSSF-36, presentaron diferencias estadística y clínicamente significativas $(12,1$ con IC 95\% = 12,01- 12,19) evidenciando la afectación negativa de la CVRS en esta población con un mayor impacto en su bienestar físico al compararse con el valor de referencia de 50 puntos establecido para poblaciones sanas (16); esto constituye un resultado relevante en miras al establecimiento de estrategias de intervención, comparación con otro tipo de enfermedades crónicas y priorización de la prestación de servicios de salud en reumatología.

El puntaje global de la funcionalidad física evaluada través del HAQ-DI (1.12) indica una afectación moderada de esta, que a su vez corrobora el resultado anteriormente expuesto sobre la afectación del bienestar físico en este grupo de pacientes, cuya relación inversa con los dominios del componente físico del MOSSF-36, se ha reportado en diversos estudios, como los de Linde et al (79), Yacoub et al (30) y Radner et al (45).

La importancia del HAQ-DI para evaluar la funcionalidad física en artritis reumatoide se evidencia en aspectos como su disponibilidad en más de 60 idiomas, el aval entregado por parte del Colegio Americano de Reumatología para su aplicación en ensayos clínicos y la gran cantidad de investigaciones en esta enfermedad que reportan su utilización $(80,81)$. En este orden de ideas, múltiples investigaciones han demostrado su asociación con aspectos determinantes en la historia natural de la enfermedad como la duración (81,82), el daño radiológico (82-84), la actividad inflamatoria (84), el riesgo de mortalidad (85) y el desarrollo de discapacidad laboral $(86,87)$; por lo que este índice de funcionalidad física se constituye como mecanismo de evaluación, seguimiento, pronóstico y remisión de la enfermedad $(7,88)$.

Por su parte, la evaluación de la CVRS en artritis reumatoide, supone un enfoque multidimensional que es inherente a instrumentos como el MOSSF-36 los cuales permiten el conocimiento del estado de salud de un individuo desde su perspectiva y del impacto que este tipo de enfermedades tienen sobre diferentes aspectos que estructuran su forma de vida y cotidianidad, emergiendo como mecanismo para la comprensión de la enfermedad desde esferas diferentes a la biológica -clínica. En este sentido, la aplicación del MOSSF-36 ha demostrado una gran relevancia y utilidad, al constituirse en un complemento de las mediciones clínicas y funcionales de la artritis reumatoide en diferentes estudios epidemiológicos $(89,90)$.

Entre las principales limitaciones de este estudio se destaca que no fue posible evaluar la heterogeneidad u homogeneidad entre los estudios individuales con pruebas como Dersimonian y Laird's, el gráfico de Galbraith o el gráfico de L'Abbé, dado que la naturaleza de los datos de las investigaciones originales no lo posibilitaron. Algo similar ocurrió con el sesgo de publicación, el cual no se pudo establecer a través del funnelplot. Por su parte el análisis de sensibilidad no fue posible realizarlo por subgrupos debido a que no se encontró un criterio común diferente de la presencia de la AR que permitiera el agrupamiento de los estudios. Pese a estas limitaciones, el estudio demostró la pertinencia de las medidas combinadas de funcionalidad física y CVRS dada su robustez en el análisis de sensibilidad $(80,81)$, a pesar de la heterogeneidad entre los resultados de los estudios individuales producto de las particularidades metodológicas y/o poblacionales de cada uno de ellos.

Finalmente los resultados de esta revisión evidencian que la funcionalidad física de las personas con artritis reumatoide se ve afectada, lo que resulta fundamental para la intervención de los enfermos, teniendo en cuenta el valor pronóstico del índice 
empleado para la discapacidad laboral y la mortalidad; de igual modo,la medición del MOSSF-36 evidenció una mayor afectación de la dimensión física, aunque no debe desconocerse el bajo puntaje en el dominio mental. Los resultados evidencian la necesidad de una intervención holística del individuo con artritis reumatoide dado que esta enfermedad impacta de forma negativa, no solo aspectos clínicos y biológicos, sino el perfil de salud física y mental percibido por el paciente.

\section{Referencias}

1. Gabriel SE. The epidemiology of rheumatoid arthritis. Rheum Dis Clin North Am 2001; 27 (2): 269-81.

2. Symmons D, Mathers C, Pfleger B. The global burden of rheumatoid arthritis in the year 2000. Geneva:World Health Organization; 2003. [Acceso: 23 Abril 2014]. Disponible en: http:// www3.who.int/whosis/menu.cfm?path=evidenceburden_ gbd2000docs\&language $=$ english .

3. Willkens RF, Nepom GT, Marks CR,Nettles JW,Nepom BS. Association of HLA-Dw16 with rheumatoid arthritis in Yakima Indians: further 1417-32 evidence for the "shared epitope" hypothesis. Arthritis Rheum. 1991; 34 (1): 43-7.

4. Martínez Sánchez FG, González Domínguez J, Amian M, Puntas MD, Salmoral A, Escudero A, et al. Estudio de prevalencia de la artritis reumatoide en un área rural. Rev Esp Reumatol. 2000;27:S179

5. Cho u CT, Pel L, Chan DM, Lee CF, Schumacher HR, Liang M. Prevalence of rheumatic diseases in Taiwan: A population study of urban, suburban, and rural differences. J Rheumatol. 1994; 21(2):302-6.

6. Delgado-Vega A, Martín J, Granados J, Anaya J. Epidemiología genética de La artritis reumatoide:¿qué esperar de américa latina? Biomédica. 2006; 26(4):562-84.

7. Sociedad española de reumatología. Actualización de la guía de práctica clínica para el manejo de la artritis reumatoide en España 2011. [Acceso: 23 Mayo 2014]. Disponible en: http://www.guiasalud.es/GPC/GPC_503_ Guipcar_\%28diciembre-2011\%29.pdf.

8. Caballero C. Artritis reumatoide como enfermedad de alto costo. Rev Colomb Reumatol. 2004; 11(3): 225:231.

9. Schneeberger E, Marengo M, Papasidero S, Chaparro R, Citera G. Clinimetría en artritis reumatoide. Rev Argent Reumatol. 2008; 19(2):8-26.

10. Sanhueza M, Castro M, Merino J. Optimizando la funcionalidad del adulto mayor a través de una estrategia de autocuidado. Av. Enferm. 2012; XXX (1): 23-31.

11. Schwartzmann L. Calidad de vida relacionada con la salud: aspectos conceptuales. Ciencia y enfermería 2003; IX (2): 9-21.
12. Patrick D, Erickson P. Health Policy, Quality of Life: Health Care Evaluation and Resource allocation. Oxford University Press. New York; 1993.

13. Portal sanitario de la región de Murcia. De Madrigal M, Velandrino A, Ruzafa M. Evaluación de Estudios de Calidad de Vida Relacionada con la Salud. . [Acceso: 24 Enero 2014] Disponible en: http://www.murciasalud.es/recursos/ ficheros/136636-capitulo_17.pdf.

14. Lillegraven S, Kvien TK. Measuring disability and quality of life in established rheumatoid arthritis. Best Pract Res Clin Rheumatol. 2007;21(5):827-40.

15. Bruce B, Fries JF. The Stanford Health Assessment Questionnaire: dimensions and practical applications. Health Qual Life Outcomes. 2003;1:20.

16. Vilagut G, Ferrera M, Rajmilb L, Rebolloc P, Permanyer-Miraldad G, Alonso J, et al. El Cuestionario de Salud SF-36 español: una década de experiencia y nuevos desarrollos. Gac Sanit. 2005; 19(2):135-50.

17. Ware JE Jr, Sherbourne CD. The MOS 36-ítem short form health survey: Conceptual framework and item selection. Med Care 1992; 30(6):473-483.

18. Feroz AHM, Islam MN, ten Klooster PM, Hasan M, Rasker JJ, Haq SA. The Bengali Short Form-36 was acceptable, reliable, and valid in patients with rheumatoid arthritis. Journal of Clinical Epidemiology 2012;65(11):1227-35.

19. Khera K, Nagappa AN, Balakrishnan R, Sharma S. PMS92 Adaptation and Validation of an Indian Version of the Health Related Quality of Life Scale (IND-HAQ) in Indian Patients with Rheumatoid Arthritis. Value in Health. 2012;15:A50-A1.

20. Koh ET, Leong KP, Tsou IY, Lim VH, Pong LY, Chong SY, Seow A. The reliability, validity and sensitivity to change of the Chinese version of SF-36 in oriental patients with rheumatoid arthritis. Rheumatology (Oxford). 2006;45(8):1023-8.

21. Linde L, Sorensen J, Ostergaard M, Horslev-Petersen $K$, Hetland ML. Health-related quality of life: validity, reliability, and responsiveness of SF-36, 15D, EQ-5D [corrected] RAQoL, and $\mathrm{HAQ}$ in patients with rheumatoid arthritis. J Rheumatol. 2008;35(8):1528-37.

22. Rugiene $R$, Dadoniene J, Venalis A. Adaptation of healthrelated quality of life ("SF-36") questionnaire, its validation and assessment of performance for control group and patients with rheumatoid arthritis. Medicina (Kaunas). 2005;41(3):232-9.

23. Ten Klooster PM, Vonkeman HE, Taal E, Siemons L, Hendriks $\mathrm{L}$, de Jong AJ, et al. Performance of the Dutch SF-36 version 2 as a measure of health-related quality of life in patients with rheumatoid arthritis. Health Qual Life Outcomes. 2013;11:77.

24. Soto Álvarez J. Incorporación de estudios de calidad de vida relacionada con la salud en los ensayos clínicos: bases y recomendaciones prácticas. Uso de una lista-guía para su correcto diseño y/o evaluación. An Med Interna. 2003; 20(12): 633-644.

25. Yamanaka H, Seto Y, Tanaka E, Furuya T, Nakajima A, Ikari K, et al. Management of rheumatoid arthritis: the 2012 perspective. Mod Rheumatol. 2013;23(1):1-7.

26. Rojas-Villarraga A, Bayona J, Zuluaga N, Mejia S, Hincapie ME, Anaya JM. The impact of rheumatoid foot on disability 
in Colombian patients with rheumatoid arthritis. BMC Musculoskelet Disord. 2009;10:67.

27.Wallenius $M$, Skomsvoll JF, Koldingsnes $W$, Rodevand $E$, Mikkelsen K, Kaufmann C, et al. Comparison of work disability and health-related quality of life between males and females with rheumatoid arthritis below the age of 45 years. Scand J Rheumatol. 2009;38(3):178-83.

28. Nagappa AN, Khera K, Balakrishnan R, Rau NR, Thunga G. PMS62 Predictors of Health Related Quality of Life in a Cohort of Patients with Rheumatoid Arthritis in Southern India. Value in Health. 2012;15(4):A45.

29. Kojima M, Kojima T, Ishiguro N, Oguchi T, Oba M, Tsuchiya $\mathrm{H}$, et al. Psychosocial factors, disease status, and quality of life in patients with rheumatoid arthritis. J Psychosom Res. 2009;67(5):425-31.

30. Ibn Yacoub Y, Amine B, Laatiris A, Hajjaj-Hassouni N. Healthrelated quality of life in Moroccan patients with rheumatoid arthritis. Clin Rheumatol. 2012;31(10):1471-7.

31. Mota LMHd, Santos Neto LLd, Burlingame RW, Ménard HA, Pereira IA, Carvalho JFd, et al. Disability and quality-of-life are not influenced by the prevalence of autoantibodies in early rheumatoid arthritis patients - results of the Brasília Cohort. Rev Bras Reumatol. 2012;52(6):819-829.

32. Nicassio PM, Kay MA, Custodio MK, Irwin MR, Olmstead R, Weisman $\mathrm{MH}$. An evaluation of a biopsychosocial framework for health-related quality of life and disability in rheumatoid arthritis. J Psychosom Res. 2011;71(2):79-85.

33. Ovayolu N, Ovayolu O, Karadag G. Health-related quality of life in ankylosing spondylitis, fibromyalgia syndrome, and rheumatoid arthritis: a comparison with a selected sample of healthy individuals. Clin Rheumatol. 2011;30(5):655-64.

34. Akar S, Can G, Binicier O, Aksu K, Akinci B, Solmaz D, et al. Quality of life in patients with Takayasu's arteritis is impaired and comparable with rheumatoid arthritis and ankylosing spondylitis patients. Clin Rheumatol. 2008;27(7):859-65.

35. García H, Lugo Agudelo L, Gomez C. Confiabilidad del cuestionario de calidad de vida en salud sf-36 en Medellín, Colombia . Rev Fac Nac de Salud Pública.2006; 24(2):37-50

36. Ware JE, Gandek B, kosinski, Aaronson NK, Apolone G,Brazier J, et al. The equivalence of SF-36 summary health scores estimated using standard and country-specific algorithms in 10 countries: results from the IQOLA project. Intenational Quality of Life Assessment. J Clin epidemiol. 1998;51(11):1167-70.

37.Urrútia G, Bonfill X. Declaración PRISMA: una propuesta para mejorar la publicación de revisiones sistemáticas y metaanálisis. Medicina Clínica. 2010;135(11):507-11.

38. Medina F, Galván M. Estudios estadísticos y prospectivos. Imputación de datos: Teoría y práctica. División Estadística y proyecciones Económicas Naciones Unidas. CEPAL. 2007 [Acceso: 4 Mayo 2014] Disponible en: www.eclac.org/ publicaciones/ xml/9/29949/LCL2772e.pdf.

39. Corbacho Ml, Dapueto JJ. Assessing the functional status and quality of life of patients with rheumatoid arthritis. Rev Bras Reumatol. 2010;50(1):31-43.

40. Courvoisier DS, Agoritsas T, Glauser J, Michaud K, Wolfe F, Cantoni $\mathrm{E}$, et al. Pain as an important predictor of psychosocial health in patients with rheumatoid arthritis. Arthritis Care Res (Hoboken). 2012;64(2):190-6.

41. Eser F, Garip Y, Bodur H. Extraarticular manifestations in Turkish patients with rheumatoid arthritis: impact of EAMs on the health-related quality of life in terms of disease activity, functional status, severity of pain, and social and emotional functioning. Rheumatol Int. 2012;32(6):1521-5.

42. Gronning K, Rodevand E, Steinsbekk A. Paid work is associated with improved health-related quality of life in patients with rheumatoid arthritis. Clin Rheumatol. 2010;29(11):1317-22.

43. Hodkinson B, Musenge $E$, Ally M, Meyer PW, Anderson R, Tikly M. Functional disability and health-related quality of life in South Africans with early rheumatoid arthritis. Scand J Rheumatol. 2012;41(5):366-74.

44. Jorge RT, Brumini C, Jones A, Natour J. Body image in patients with rheumatoid arthritis. Mod Rheumatol. 2010;20(5):491-5.

45. Radner H, Smolen JS, Aletaha D. Comorbidity affects all domains of physical function and quality of life in patients with rheumat oid arthritis. Rheumatology (Oxford). 2011;50(2):381-8.

46. Tang B, Naim A, McKenzie RS, Bailey R, Freedman D, Wagner S, et al. PMS39 impairments in quality of life, daily function, and work productivity and activity in rheumatoid arthritis patients who use subcutaneous biologic therapies. Value in Health. 2009;12(3):A70-A1.

47.Thyberg I, Skogh T, Hass UA, Gerdle B. Recent-onset rheumatoid arthritis: a 1-year observational study of correlations between health-related quality of life and clinical/laboratory data. J Rehabil Med. 2005;37(3):159-65.

48. Ibn Yacoub $Y$, Amine B, Laatiris A, Hajjaj-Hassouni N. Spinsterhood and its impact on disease features in women with rheumatoid arthritis . Health Qual Life Outcomes. 2011;9:58.

49. Blaizot A, Monsarrat $P$, Constantin A, Vergnes JN, de Grado GF, Nabet $C$, et al. Oral health-related quality of life among outpatients with rheumatoid arthritis. Int Dent J. 2013;63(3):145-53.

50. Carreño A, Fernández I, Badia X, Varela C, Roset M. Using HAQDI to estimate HUI-3 and EQ-5D utility values for patients with rheumatoid arthritis in Spain. Value in Health. 2011;14(1):192200.

51. Garip Y, Eser F, Bodur H. Health-related quality of life in rheumatoid arthritis: comparison of RAQoL with ot her scales in terms of disease activity, severity of pain, and functional status. Rheumatol Int. 2011;31(6):769-72.

52. Haroon N, Aggarwal A, Lawrence A, Agarwal V, Misra R. Impact of rheumatoid arthritis on quality of life. Mod Rheumatol 2007;17(4):290-5.

53. Helland $Y$, Dagfinrud $H$, Kvien TK. Perceived influence of health status on sexual activity in RA patients: associations with demographic and disease-related variables. Scand J Rheumatol. 2008;37(3):194-9

54. Khera K, Nagappa AN, Thunga G, Sam K. PMS9 health-related quality of life in indian patients with rheumatoid arthritis. Value in Health. 2010;13(7):A559.

55. Kobelt G, Woronoff AS, Richard B, Peeters P, Sany J. Disease status, costs and quality of life of patients with rheumatoid arthritis in France: the ECO-PR Study. Joint Bone Spine. 2008;75(4):408-15. 
56. Marra CA, Lynd LD, Esdaile JM, Kopec J, Anis AH. The impact of low family income on self-reported health outcomes in patients with rheumatoid arthritis within a publicly funded health-care environment. Rheumatology (Oxford). 2004;43(11):1390-7.

57.Pentek M, Szekanecz Z, Czirjak L, Poor G, Rojkovich B, Polgar $A$, et al. Impact of disease progression on health status, quality of life and costs in rheumatoid arthritis in Hungary. Orv Hetil. 2008;149(16):733-41.

58. Standfield L, Norris S, Harvey C, Elliot L, Riordan J, Hall S, et al. Relationship between rheumatoid arthritis disease severity, health-related utility, and resource use in australian patients: A cross-sectional, multicenter study. Clinical Therapeutics. 2010;32(7):1329-42.

59. Tang CH, Hsu PN, Hsu JY, Fang CH. PMS77 - Health-State Utilities in Measuring Health-Related Quality of Life Among Patients with Rheumatoid Arthritis in Taiwan. Value in Health. 2013;16(7):A568.

60. Birtane M, Uzunca K, Tastekin N, Tuna H. The evaluation of quality of life in fibromyalgia syndrome: a comparison with rheumatoid arthritis by using SF-36 Health Survey. Clin Rheumatol. 2007;26(5):679-84.

61. Garcia-Poma A, Segami MI, Mora CS, Ugarte MF, Terrazas $H N$, Rhor EA, et al. Obesity is independently associated with impaired quality of life in patients with rheumatoid arthritis. Clin Rheumatol. 2007;26(11):1831-5.

62. He DY, Yao C, Zhao N, Ning Y, Han C. PMS23 Health Related Quality of Life, Measured by the Short Form-36, of Patients with Rheumatoid Arthritis and Ankylosing Spondylitis in an Urban Population of China. Value in Health. 2012;15(7):A675.

63. Ho RC, Fu EH, Chua AN, Cheak AA, Mak A. Clinical and psychosocial factors associated with depression and anxiety in Singaporean patients with rheumatoid arthritis. Int J Rheum Dis. 2011;14(1):37-47.

64. Kanecki K, Tyszko P, Wislowska M, Lyczkowska-Piotrowska J. Preliminary report on a study of health-relat ed quality of life in patients with rheumatoid arthritis. Rheumatol Int. 2013;33(2):429-34.

65. Kavati A, Rappaport H. PMS53 - Impact Of Rheumatoid Arthritis ( $\mathrm{Ra}$ ) On Quality Of Life (Qol) In A Nationally Representative Population In The United States. Value in Health. 2013;16(3):A227.

66. Kothe R, Kohlmann T, Klink T, Ruther W, Klinger R. Impact of low back pain on functional limitations, depressed mood and quality of life in patients with rheumatoid arthritis. Pain. 2007;127(12):103-8

67.Lempp H, Ibrahim F, Shaw T, Hofmann D, Graves H, Thornicroft $\mathrm{G}$, et al. Comparative quality of life in patients with depression and rheumatoid arthritis. Int Rev Psychiatry. 2011;23(1):118-24.

68. Picavet HS, Hoeymans N. Health related quality of life in multiple musculoskeletal diseases: SF-36 and EQ-5D in the DMC3 study. Ann Rheum Dis. 2004;63(6):723-9.

69. Piccinni A, Maser JD, Bazzichi L, Rucci P, Vivarelli L, Del Debbio A, et al. Clinical significance of lifetime mood and panic-agoraphobic spectrum symptoms on quality of life of patients with rheumatoid arthritis. Comprehensive Psychiatry. 2006;47(3):201-8.
70. Rupp I, Boshuizen HC, Jacobi CE, Dinant HJ, van den Bos GA. Impact of fatigue on health-related quality of life in rheumatoid arthritis. Arthritis Rheum. 2004;51(4):578-85.

71. Salaffi F, Carotti M, Gasparini S, Intorcia M, Grassi W. The health-related quality of life in rheumatoid arthritis, ankylosing spondylitis, and psoriatic arthritis: a comparison with a selected sample of healthy people. Health Qual Life Outcomes. 2009;7:25.

72. Salaffi F, Sarzi-Puttini P, Girolimetti R, Atzeni F, Gasparini S, Grassi W. Health-related quality of life in fibromyalgia patients: a comparison with rheumatoid arthritis patients and the general population using the SF-36 health survey . Clin Exp Rheumatol. 2009;27(5 Suppl 56):S67-74.

73. Tander B, Cengiz K, Alayli G, Ilhanli I, Canbaz S, Canturk F. A comparative evaluation of health related quality of life and depression in patients with fibromyalgia syndrome and rheumatoid arthritis. Rheumatol Int. 2008; 28(9):859-65.

74. Tang B, Rahman MI, Annunziata K, Thompson H, Nairn A, Freedman D. PMS25 Association between out-of-pocket expenses and clinical outcomes. and quality of life in patients with rheumatoid arthritis. Value in Health. 2008;11(3):A262.

75. Vinaccia S, Fernández H, Moreno San Pedro E, Padilla GV. Spanish Quality of Life Measure for Rheumatoid Arthritis (QOL-RA) Tested in Colombia. Rev Colomb Reumatol.2006;13(4)264-70.

76. West $E$, Jonsson SW. Health-related quality of life in rheumatoid arthritis in Northern Sweden: a comparison between patients with early RA, patients with medium-term disease and controls, using SF-36. Clin Rheumatol. 2005;24 (2):117-22.

77. West $E$, Wållberg-Jonsson S. Health-related quality of life in Swedish men and women with early rheumatoid arthritis. Gender Medicine. 2009;6(4):544-54.

78. Lubeck D. Patient-Reported Outcomes and Their Role in the Assessment of Rheumatoid Arthritis. Pharmacoeconomics. 2004; 22 Suppl. 1: 27-38

79. Linde, L., Sørensen, J., ØSTERGAARD, M., Hørslev-Petersen, K., \& Hetland, M. L. Health-related quality of life: validity, reliability, and responsiveness of SF-36, EQ-15D, EQ-5D, RAQoL, and HAQ in patients with rheumatoid arthritis. The Journal of rheumatology.2008;35(8): 1528-37.

80. Felson DT, Anderson JJ, Boers M, Bombardier C, Chernoff $M$, Fried $B$, et al. The American College of Rheumatology preliminary core set of disease activity measures for rheumatoid arthritis clinical trials. The Committee on Outcome Measures in Rheumatoid Arthritis Clinical Trials. Arthritis Rheum. 1993; 36(6):729-40.

81. Bruce B and Fries JF: The Stanford health assessment questionnaire (HAQ): a review of its history, issues, progress, and documentation J Rheumatol. 2003; 30(1):167-78.

82. Scott DL, Pugner K, Kaarela K, Doyle DV, Woolf A, Holmes J, et al. The links between joint damage and disability in rheumatoid arthritis. Rheumatology. 2000;39(2):122-132.

83. Schneeberger EE, Citera G, Maldonado Cocco JA, Salcedo M, Chiardola F, Arriola MS, et al. Características del daño radiológico y principales factores asociados en una cohorte de pacientes con artritis reumatoide. Rev Argent Reumatol 2005;16 (Supl 1):13. 
84. Rupp I, Boshuizen HC, Dinant HJ, Jacobi CE, van den Bos GA. Disability and health-related quality of life among patients with rheumatoid arthritis: association with radiographic joint damage, disease activity, pain, and depressive symptoms. Scand J Rheumatol. 2006;35(3):175-81.

85. Michaud K, Vera-Llonch M, Oster G. Mortality risk by functional status and health-related quality of life in patients with rheumatoid arthritis. J Rheumatol. 2012;39(1):54-9.

86. Sokka T, Pincus T. Markers for work disability in rheumatoid arthitis. J Rheumatol. 2001; 28(7):1718-22.

87. De Croon EM, Sluiter JK, Nijssen TF, Dijkmans BAC, Lankhorst GJ, Frings-Dresen MHW. Predictive factors of work disability in rheumatoid arthritis: a systematic literature review. Ann Rheum Dis. 2004; 63(11):1362-1367.

88. Robustillo M, Rodríguez J, ¿Son útiles los factores pronóstico en la artritis reumatoide? Reumatol Clin. 2011;7(5):339-342.

89. Matcham F, Scott I, Rayner L, Hotopf M, Kingsley G, Norton $S$, et al The impact of rheumatoid arthritis on quality-of-life assessed using the SF-36: A systematic review and metaanalysis. Seminars in Arthritis and Rheumatism. 2014 Article in press

90. Birrell F, Hassell B, Jones P, Dawes P. How does the short form 36 health questionnaire (SF-36) in rheumatoid arthritis (RA) relate to RA outcome measures and SF-36 population values? A crosssectional study. Clinical rheumatology, 2000; 19(3), 195-199.

91. Sociedad española de bioquímica clínica y patología molecular. [Acceso: 13 Agosto 2014].Metaanálisis. Disponible en: http:// www.seqc.es/dl.asp?.pdf.

92. Díaz S, Fernández S. Revisiones sistemáticas y Metaanálisis (II). [Acceso: 10 Agosto 2014] Disponible en http://www.fisterra. com/mbe/investiga/metaanalisis/RSyMetaanalisis2.asp

\section{Opina sobre este artículo:}

\section{8 in $8+S$}

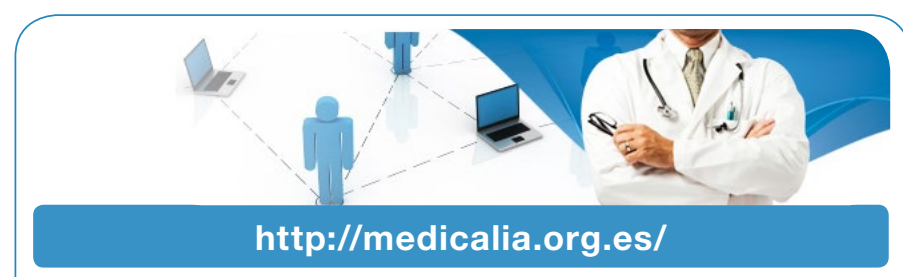

Los médicos disponen de una red social para intercambiar experiencias clínicas, comentar casos y compartir conocimiento. También proporciona acceso gratuíto a numerosas publicaciones. ¡Únase ahora!

\section{Publique con iMedPub}

\section{http://www.imed.pub}

\section{Biomedicina}

$\checkmark$ Biomedicina es la primera revista de acceso abierto en Español, dedicada a todas las áreas de la medicina.

$\checkmark$ Recoge artículos de investigación sobre todas las áreas de la medicina. 